\title{
New use heat transfer theories for the design of heat setting machines for precise post-treatment of dyed fabrics
}

\author{
Ralph W.L. Ip $p^{1, a}$, Wan lok Cheong ${ }^{2, b}$ \\ ${ }^{1,2}$ Department of Mechanical Engineering, The University of Hong Kong, Pokfulam Road, Hong Kong \\ SAR \\ aralphip@hkucc.hku.hk, ${ }^{\text {bh0605993@hku.hk }}$
}

Keywords: heat-setting machine; drying rate; capillary flow; mass flux

\begin{abstract}
Fabrics are needed further treatment after dyeing to restore their original mechanical properties by suitable drying/shrinkage process because of wetted and elongated fabrics cannot be used for clothes making. Heating up the dyed fabrics at suitable temperature can restore their original shapes and geometries by releasing the internal stress introduced by dyeing process. Thus, heat setting is a commonly used post-treatment process to stabilize fabric geometrical dimensions and prevent further shrinkage. Hot air jet impingement[1] and moist heat are conventional drying methods for different applications. Despite the well establishments of these drying technologies, most of the applications are for materials like clay and paper, and few on the study of textile materials. In fact that most of the developed heat setting machines used in textile industry are only designed by empirical models and lack of theoretical bases. This situation will obstruct further improvement of the drying technology. In this paper, a theoretical basis heat transfer model is developed for a precise description of a heated air flowing process for heat setting machine design. In the machine design, a better airflow circulation strategy for an efficient drying is addressed. Equations for heat and mass transfer in moist porous materials and theories on thermo- and fluid-dynamics are used to support the machine design. Outcomes from the research are to develop a heat transfer model that provides more precise and effective calculation for heat setting machine design that unavailable from the developed machine prototypes.
\end{abstract}

\section{The operations of fabric drying on heat setting machines}

A stream of heated air blows on both sides of the wet fabric in the drying process. The conveyor carries the fabric into the machine chamber. Heated air blows from the top and bottom rows of nozzles, which are offset to each other. The fabric forms a wave pattern when air blows on it. Water will leave the fabric by evaporation when enough amount of latent heat is absorbed. A humidity controller is used to control the air temperature to maintain a precise drying in the process. Steam is used to heat up the air by indirect contact through a coil type heat exchanger.

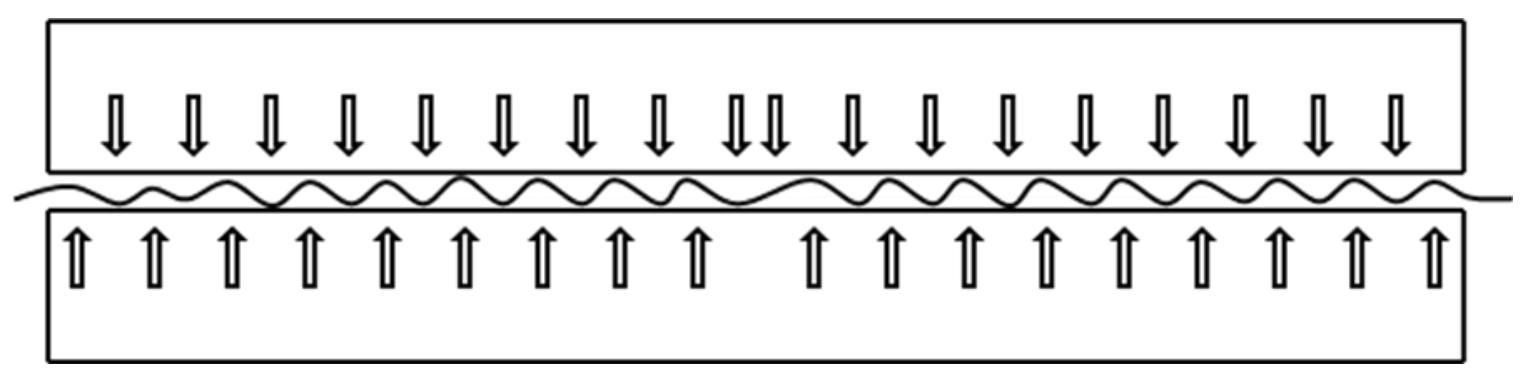

Fig. 1. Nozzle design with wave pattern fabrics 


\section{The model of heated air drying}

In a heated air drying process, two periods of drying are commonly observed, they are "Constant drying rate" and "Falling drying rate"[2]. The constant drying period has a liner relationship of the moisture percentage and drying time. At the end of this period, the drying characteristics will move to the second period. In the falling drying period, a non-linear relationship is found for moisture percentage and drying time. The point of switching of the period is called "Critical point of drying". This is illustrated in Fig.2.

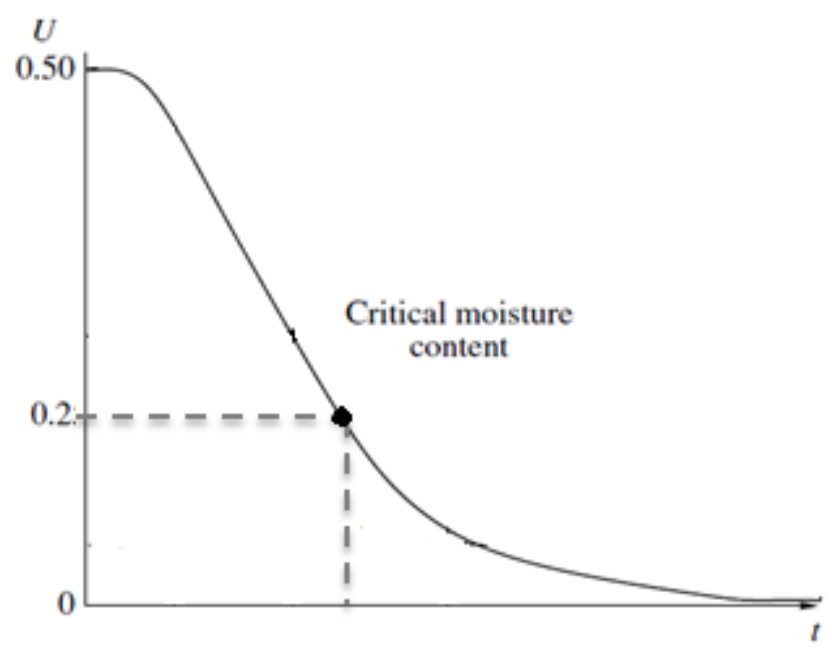

In micro-level[3], free water on the fabric surface is removed by convection Fig. 2. Typical drying curve heat transfer mechanism. The fabric temperature keeps constant, and the fabric surface remains wet until the critical point of drying is reached. In the falling drying rate period, temperature of fabric continuously increases up to temperature of the heated air. The moisture in the fabric capillary-pores is in funicular states, meniscuses are observed. The meniscuses can only found in larger size capillaries at initial stage, but gradually found in capillaries of smaller size. It causes a resistance to the diffusion path for the water molecules, thus, the drying rate decreases until the end of drying.

\section{Approaches to mathematical analysis of drying}

Boundary conditions for the constant drying rate period. In the first period of drying, the amount of water evaporated to vapour inside the fabric is much smaller than that on the boundary surface. Thus, assuming that the fluxes of vapour and air are negligible in this period.

$\mathrm{W}^{\mathrm{v}}=0$ and $\mathrm{W}^{\mathrm{a}}=0$

Then, the mass balance equation for moisture content calculation in a drying process is given as:

$\rho^{\mathrm{S}} \mathrm{X}^{1}=-\operatorname{div} \mathrm{W}^{1}+\psi^{1}$

$\rho^{\mathrm{S}} \mathrm{X}^{\mathrm{v}}=\psi^{\mathrm{v}}<<\rho^{\mathrm{S}} \mathrm{X}^{\mathrm{l}}$

$\rho^{\mathrm{S}} \mathrm{X}^{\mathrm{a}}=0$

where $\rho$ is mass density, $X$ is mass content, $W$ is mass flux, $\psi$ is rate of phase transition,

$\mathrm{S}$ is entropy, 1 is liquid phase, $\mathrm{v}$ is vapour phase and a is gas phase

Equation (1) shows phase transition of water inside the fabric

Equations (2) and (3) shows phase transition of vapour and air inside the fabric that is kept constant 
Boundary conditions for the falling drying rate period. Although menisci start to recede into the fabric, the top surface does not become completely dry. The liquid efflux from the fabric becomes weaker. The moisture transport in this period is rather complex, and the characteristics can be divided into hygroscopic and non-hygroscopic fabrics, as shown in Fig. 2.
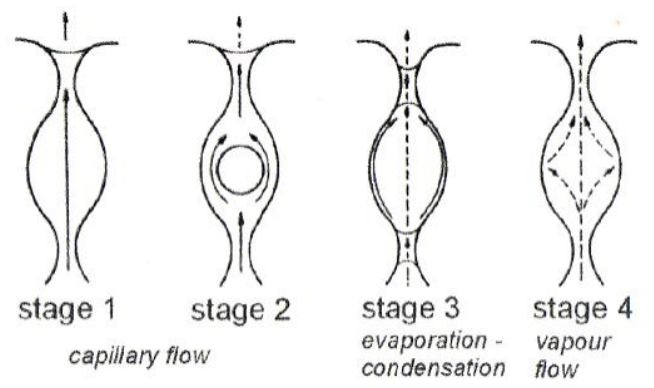

DRYING

Fig. 3. Illustration of conditions inside fabrics during falling drying rate period

- At the beginning of the falling drying rate period, fabric is completely saturated and moisture flows in the form of liquid fluxes mainly due to capillary uplift as illustrated in stage 1.

- Air pockets gradually formed at stage 2 to replace some of the moisture, the water flows outside due to the gradient of capillary potential.

- With further drying, moisture content decreases and water bridges particles in solid skeleton as shown in stage 3 .

- Finally, drying takes place in all inner portions of fabric. The partial pressure of water vapour decreases.

The mass balance equations for the falling drying rate period are given as:

$\rho^{\mathrm{S}} \mathrm{X}^{1}=\psi^{\mathrm{a}}$

$\rho^{\mathrm{S}} \mathrm{X}^{\mathrm{v}}=-\operatorname{div} \mathrm{W}^{\mathrm{v}}+\psi^{\mathrm{a}}$

$\rho^{\mathrm{S}} \mathrm{X}^{\mathrm{a}}=-\operatorname{div} \mathrm{W}^{\mathrm{a}}$

Determination of coefficients for mass fluxes in drying

The mass flux equation for the constant drying rate period is:

$$
\begin{aligned}
& W^{l}=-\wedge^{l}\left[\left(\frac{\partial \mu^{l}}{\partial T}\right) T-\frac{\gamma^{l}}{\rho^{s}} \varepsilon+\left(\frac{\partial \mu^{l}}{\partial \theta^{l}}\right) \theta^{l}-g\right] \\
& C^{l}=\left(\frac{\partial \mu^{l}}{\partial \theta^{l}}\right)
\end{aligned}
$$

Where $\wedge$ is coefficient of mass transfer, $\mu$ is chemical potential, $\theta$ is relative moisture content, $\mathrm{T}$ is temperature and $\mathrm{g}$ is gravity

The flow of condensate phase in fabric is mainly due to capillary forces and gravitational force in the case of relatively large fibre pores in the first drying period. $C^{1}$ is a material coefficient related to the moisture cohesive force in the fabric.

The mass flux equation for the falling drying rate period is: 
$W^{a}=-\wedge^{a}\left[\left(\frac{\partial \mu^{a}}{\partial T}\right) T+\sum\left(\frac{\partial \mu^{a}}{\partial \theta^{v}}\right) \theta^{v}\right]$

The vapour is produced inside the fabric due to phase transition of water into vapour, thus, the efflux of vapour is significant.

The coefficient $\mathrm{C}$ for a vapour may be experimentally measured.

\section{Experimental studies of fabric drying and coefficient determination}

Three sets of data were collected from the test of moisture contents in fabrics under heated air drying at different temperature. The collected data are then plotted and fitted by trend lines. Linear and second order equations are determined from the trend lines, and the coefficient of mass transfer stated in Section 4 can be determined.

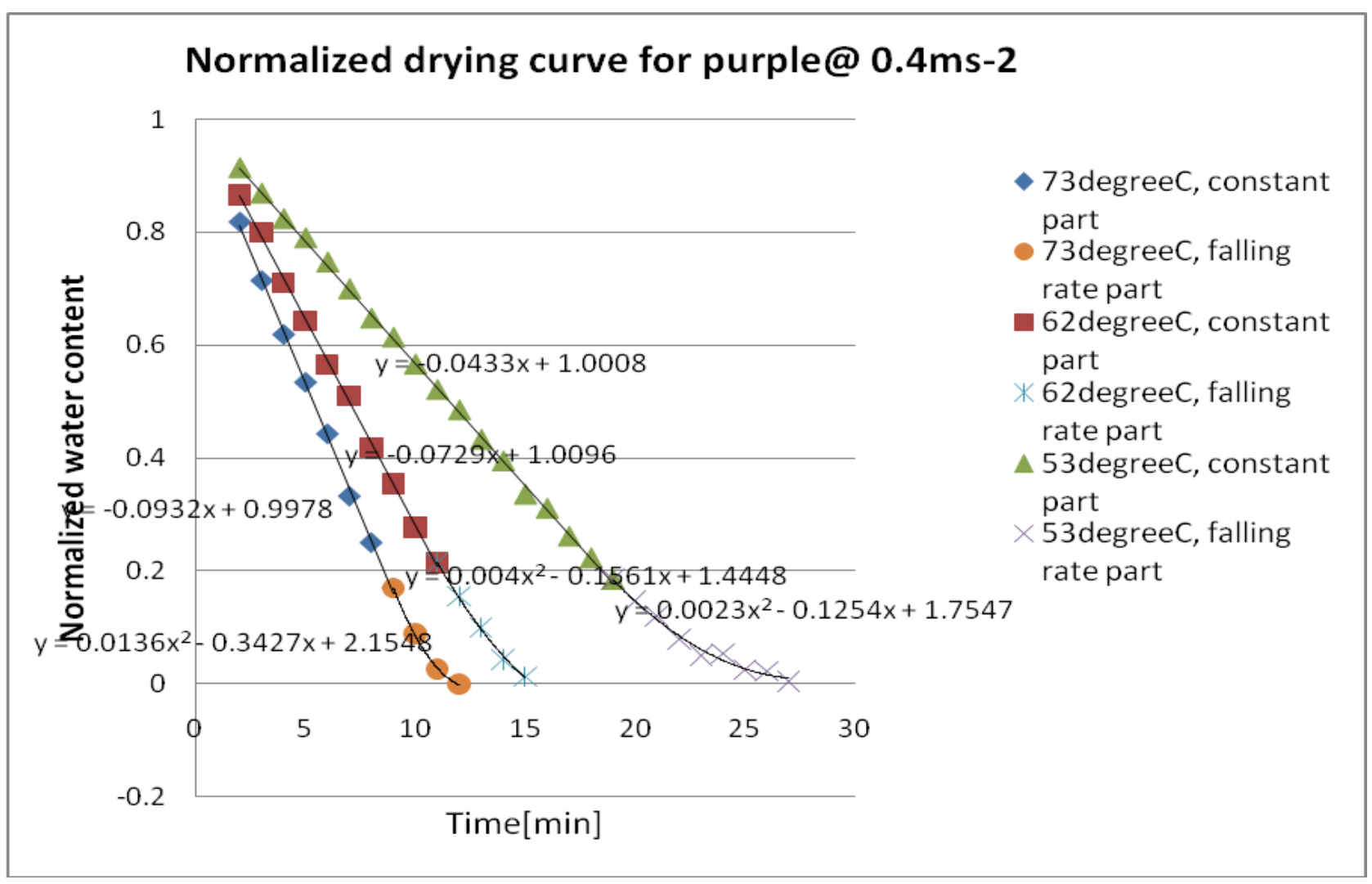

Fig. 4. A sample result on the experimental studies of fabric drying

\section{Conclusions}

Principles of water movement due to phase change in heated air drying have been studied. Equations of mass transfer between water, vapour and air in the drying process have been shown. In the calculation, the coefficients in the equations should be experimental determined. The research provides a new study of moisture content calculation in drying instead of the traditional heat transfer calculation. More research can be done to apply the new study onto the design of heat setting machine for textile industry. 


\section{References}

[1] Nicholas D. Francis and William J.Wepfer, Jet impingement drying of a moist porous solid. Int. J. Heat Mass Transfer, Vol. 39(9)(1996), p. 1911-1923.

[2] A.K. Haghi, Transport Phenomena in Porous Media: A Review. Theoretical Foundations of Chemical Engineering, Vol.40(1) (2006), p. 14-26.

[3] J.C. Slattery, Momentum, energy, and mass transfer in contimua, McGraw Hill(1972) 